\title{
Video Article \\ A Modified Trier Social Stress Test for Vulnerable Mexican American Adolescents
}

\author{
Megan M. Johnson ${ }^{1}$, Julianna Deardorff ${ }^{1}$, Kimberly Parra ${ }^{1}$, Abbey Alkon $^{2}$, Brenda Eskenazi ${ }^{1}$, Elizabeth Shirtcliff ${ }^{3}$ \\ ${ }^{1}$ Center for Environmental Research and Children's Health $(\mathrm{CERCH})$, Berkley School of Public Health, University of California, Berkeley \\ ${ }^{2}$ San Francisco (UCSF) School of Nursing, University of California, San Francisco \\ ${ }^{3}$ Human Development and Family Studies, lowa State University
}

Correspondence to: Megan M. Johnson at meganjohnson@berkeley.edu

URL: https://www.jove.com/video/55393

DOI: doi:10.3791/55393

Keywords: Developmental Biology, Issue 125, TSST, stress, youth, adolescents, cortisol, Mexican American

Date Published: 7/10/2017

Citation: Johnson, M.M., Deardorff, J., Parra, K., Alkon, A., Eskenazi, B., Shirtcliff, E. A Modified Trier Social Stress Test for Vulnerable Mexican American Adolescents. J. Vis. Exp. (125), e55393, doi:10.3791/55393 (2017).

\section{Abstract}

The Trier Social Stress Test (TSST) is a well validated and widely used social stressor that has been shown to induce a 2-4 fold increase in cortisol, the biological output produced by the Hypothalamic-Pituitary-Adrenal (HPA) axis in humans. While studies have explored how modifications to the TSST influence stress responsivity, few studies have created a modified TSST appropriate for vulnerable youth that elicits a significant cortisol stress response. Thus, the current study sought to modify or adjust the TSST in a culturally sensitive way for a vulnerable sample of 14 year-old adolescents. The present study took place within the context of a longitudinal birth cohort study of Mexican American families in California called the Center for the Health Assessment of Mothers and Children of Salinas (CHAMACOS). The CHAMACOS sample was optimal to test the effectiveness of a modified culturally appropriate TSST, as it is comprised of Mexican American youth, who are often excluded from research. These youths also have experienced significant early life adversity. Example modifications included timed prompts, alternative math tasks, use of same-ethnicity peers as confederates, debriefing immediately after the conclusion of the TSST, and using an unknown youth examiner to administer the debrief. Saliva samples were collected at baseline (after a resting phase), and then again at 15, 30 , and $45 \mathrm{~min}$ post-TSST onset to assess cortisol concentration. A pilot study of 50 participants ( $50 \%$ female) have been analyzed for cortisol reaction to the TSST. Results confirmed that this modified version of the TSST was successful at eliciting a significant cortisol reaction, with a wide range of variability likely due to individual differences. Goals for modifications and ethnicity considerations are discussed. This study provides the foundation for future research to utilize a modified TSST with vulnerable youth.

\section{Video Link}

The video component of this article can be found at https://www.jove.com/video/55393/

\section{Introduction}

The Trier Social Stress Test (TSST) ${ }^{1}$ has become the gold standard for inducing stress in laboratory settings ${ }^{2}$. It is a well-validated and widely used social stressor that has been shown to induce a 2-4 fold increase in cortisol, the biological output produced by the Hypothalamic-PituitaryAdrenal (HPA) axis ${ }^{1,3}$. A meta-analysis concluded that two key aspects are integral to inducing the most robust HPA reactivity across the lifespan: unpredictability/uncontrollability and social evaluative threat (SET), both of which characterize the TSST ${ }^{3}$.

Adolescence may be a key period of time when SET is especially stressful, marked by a sensitive period of development, whereby social interactions facilitate learning ${ }^{4}$. For example, during early puberty and adolescence, integration with peer groups becomes motivationally salient ${ }^{5}$. Rapid increases in hormone levels at the start of puberty influence development of limbic circuits, which leads to increases in the processing of status-relevant social stimuli (i.e., admiration) as having increased motivational salience ${ }^{6}$. This increase in social affective engagement not only influences motivations and behaviors in the moment but also influences patterns of behavior and motivational learning over long intervals. Over time, this change in prioritizing social incentives can lead to health exploration and risk taking, which can promote social and affective learning and skills that underpin adult social functioning. Thus, it is no surprise that aspects of social functioning involved in the TSST speech phase are a potent form of stress for adolescents, i.e., the "good friend" topic ${ }^{7,8,9}$. However, individuals sensitized by a history of trauma or stressful life events may be primed to react poorly to socially evaluative or unpredictable situations due to poor emotional control or augmented sensitivity to stress, and this pattern of negative response could contribute to negative healthy outcomes, such as drug use or dangerous thrill seeking. Thus, understanding biological responses to SET is of utmost importance in vulnerable youth.

A handful of studies have explored how variations of SET and uncontrollability influence stress responsivity. SET can be increased or decreased based on the number of confederate judges. The TSST calls for 2 confederates, but it is more effective with four ${ }^{10}$, and less effective with 1 2 confederates $^{11,12,13}$ or with a virtual or distant audience ${ }^{14,15,16}$. Adding more negative commentary increases SET and responder rates ${ }^{11,17}$, whereas positive commentary reduces SET and responder rates ${ }^{18}$. Rates of responsivity are influenced by changing uncontrollability by (a) modifying the length of time participants have to prepare the speech ${ }^{14}$ (b) increasing participants' psychosocial resources ${ }^{19,20,21}$, or $(\mathrm{c})$ increasing participants' self-confidence prior to the $\mathrm{TSST}^{22}$. Modifications that enhance emotion suppression ${ }^{23}$, vigilance or attention to the stressor ${ }^{24,25}$, or 
thoughts about the self ${ }^{26}$ all increase TSST responsivity. Finally, the sex and age of confederate can influence stress reactivity to SET ${ }^{27,28}$. These studies illustrate that responsivity rates can be experimentally increased or decreased. In the current study, the TSST was modified by including timed prompts, alternative math tasks, use of same-ethnicity peers as confederates, debriefing immediately after the conclusion of the TSST, and using an unknown youth examiner to administer the debrief.

A recent review of stressor paradigms demonstrated particularly robust effects of social evaluative threat on cortisol elevation (HPA response) during middle adolescence ${ }^{29}$. However, there are valid concerns about using the TSST with vulnerable youth. Specifically, human subjects' protection concerns may be raised to ensure that the benefits outweigh the risks, as there is the potential trauma associated with enduring the TSST when youth are already vulnerable (e.g., coming from environments of poverty and early life adversity). Thus, there is the need to ensure that the youth do not leave in a state of elevated anxiety or agitation. However, modifications to the TSST to alleviate/mitigate some of these concerns must not reduce the overall efficacy or validity of the stressor. For example, prior studies have documented the effects of modified versions of the TSST for vulnerable youth but have had limited success in terms of provoking a rise in cortisol ${ }^{30,31}$. Further, modifications may need to be made to make the TSST more culturally sensitive and appropriate. For example, there is literature that suggests interacting with individuals of different races can induce stress and threat reactions ${ }^{32}$. Differing the racial makeup of the participant and TSST committee has been shown not to impact cortisol reactivity to the TSST ${ }^{33}$, but rather to impact cortisol recovery from the TSST ${ }^{34}$. Thus, using same-race/ethnic confederates for the TSST may eliminate any unnecessary confound of the confederates' race on the perceived stereotype threat and cortisol recovery from the TSST.

The present study took place within the context of a longitudinal birth cohort study of Mexican American families in California called the Center for the Health Assessment of Mothers and Children of Salinas (CHAMACOS). This sample is characterized as highly vulnerable due to high rates of poverty ( $76 \%$ of the families were at or below the Federal Poverty level) and high numbers of adverse early life events (mean number of lifetime events $=4.5$ for the sample, $S D=3.3$ ). In comparison, a large retrospective study conducted by a partnership between the U.S. Centers for Disease Control and Prevention and Kaiser Permanente based on thousands or respondents found only $13 \%$ to have reported 4 or more adverse childhood events ${ }^{35}$.

The TSST was administered to the youth in this study as part of a large NIH grant aimed at researching the link between adverse early life events, biological reactivity to social evaluative threat at age 14 (when the TSST was administered), and risk-taking behaviors (at age 16; data collection is still ongoing; grant1R01DA035300). During the early stages of piloting the TSST, we observed that the approach needed to be shifted to become contextually sensitive to the unique needs of this population and to mitigate poor responses (i.e., crying, becoming angry, disengaging from the TSST). To be precise, we had a $20 \%$ rate of poor behavioral responses to the TSST prior to modifications (i.e., 4 of 20 TSST participants either cried or responded with anger), whereas only $5 \%$ responded with poor responses after the modifications were implemented. Study personnel were also keenly aware of the importance of retaining youth in this longitudinal study. It should be noted that during the pilot phase, individuals who responded to the TSST with extreme stress reactions behaviorally during the debrief were, at the biological level, "non-responders," i.e., they did not demonstrate a cortisol rise. This emotional/biological juxtaposition from these initial results also highlighted the need to modify the TSST to create the optimal conditions to induce an appropriate biological response to the TSST. Thus, this study provides preliminary evidence from a subsample of youth of the efficacy of the modified TSST to induce cortisol reactivity.

\section{Protocol}

This study was approved by the Institutional Review Board from the University of California, Berkeley and the University of California, San Francisco. Participants were scheduled to arrive at the lab between 4:30-5:00 PM.

\section{Rinse Mouth}

1. Ask the participant to go to the restroom and rinse out their mouth with water only. Have the participant swish with cold water for $5-10 \mathrm{~s}$ and spit in the sink. Do this only once.

2. Say to participant, "This is to make sure there's no food or crumbs in your teeth for saliva collection."

\section{Resting Period}

1. Usher participant into a solitary room and allow them to sit for at least $10 \mathrm{~min}$. Have the participant complete non-invasive or non-provoking questionnaires such as a contact information sheet and daily living questionnaires, as well as watch a 3 min peaceful video of sea life.

\section{Saliva collection \#1 (Pre-stress)}

1. Place a line to indicate $1 \mathrm{~mL}$ of saliva on a $2 \mathrm{~mL}$ cryovial, and tell the participant to fill the cryovial to this line. Collect participant's saliva via passive drool through a two-inch straw into the $2 \mathrm{~mL}$ cryovial ${ }^{36}$ and freeze immediately $\left(-80^{\circ} \mathrm{C}\right)$.

2. Say to participant, "I will ask you to fill this vial with saliva right up to this line. The way to do it is to let saliva collect in your mouth and then let it run through the straw into the vial. It's best if you don't spit because that gets bubbles in your sample - just let the saliva run through the straw. It will help if you don't talk while you're doing this. Please try not to cough up phlegm, we just want normal saliva. Keep an eye on your saliva vial and how full it is. If there are bubbles at the top of your saliva, they should go over the line, so there is just liquid saliva right up to the line. Tell me when it fills up to the line. Here are some pictures of food posted on the wall (POINT OUT PICTURES), since thinking of favorite foods makes peoples' mouths water."

3. Offer water after the participant has completed the saliva sample. Do not allow the participant to keep the water; take it back when he/she is finished. 


\section{Preparation Period}

1. Say to participant: "In a few min, we would like you to give a five-minute speech about how you are a good friend. There is a committee of two trained reviewers who are in the room next door who are going to be listening to your speech. Your job is to make the reviewers believe that you are a good friend. They want you to explain what things make you a good friend to others. You are competing against a lot of other people for how good you are at giving your speech and convincing people that you are a good friend. The reviewers are experts in judging facial expression and body movement, and they are going to compare your speech to other people in the study. You may notice that the reviewers are taking notes. This is so we can review their notes at a later time. You will also be videotaped so that we can rate your performance later.

You'll have 3 min now to prepare for your speech. Remember, your speech should explain what it is about you that makes you a good friend, and you are competing against other people for how believable you are. After that, I will take you to the next room, where you will introduce yourself to the reviewers and give your 5-minute speech. There will be another task that comes after the speech, but the reviewers will let you know what the task is after the speech. Do you have any questions? You may write notes if you want, but you won't be able to take them into the room with you. Ok, please start preparing. I'll return in a few minutes."

2. Hand participant paper, pen, and clipboard to allow them to take notes.

3. Leave the participant alone in the room.

4. Wait $3 \mathrm{~min}$ and then come back in the room.

\section{TSST Set Up}

1. Say to participant, "Okay, it's time to go next door and get started." If the participant has written notes, take them from the participant. Say, "I can take those from you now. Thanks."

2. Walk the participant to TSST room and indicate that they should face the confederates and the video camera

3. Start the video recording before the participant enters the room. Record the entire speech and math tasks (approximately 10 min total).

4. Have the Youth Interviewer introduce the committee. "This is_(participant's name)_._(Participant)_, do you know either of these people?"

1. If the participant answers no, have the Youth Interviewer says "Okay, you can get started."

2. If the participant answers yes, have the Participant indicate whom s/he knows, and have the Youth Interviewer say "Okay, _(confederate)_, please excuse yourself. The judges need to be people who don't know you."

\section{TSST Speech Task}

1. Have Confederate 1 say, "Please tell us why you are a good friend. Begin speaking now."

2. Set the timer for $5 \mathrm{~min}$. Start timer.

3. After $\mathbf{3 0} \mathrm{s}$ of silence, use one of the following prompts to keep the participant talking. Use prompts in order each time there is a $\mathbf{3 0} \mathrm{s}$ pause.

1. Say "You still have time. Please continue."

2. Say "Why else are you a good friend?"

3. Say "Please give an example of when you were a good friend."

4. Say "Be as creative as you like. Why else are you a good friend?"

5. After all prompts above have been used, continue prompting thereafter with, "You still have time. Please continue."

4. After 5 min, have Confederate 1 say: "Please stop. Your time is up." (Proceed to math task.)

\section{TSST Math Task}

1. Have Confederate 1 say, "We are now going to ask you to count out loud backward. Start at 423, and count backward by 7's. Keep counting backward by 7's out loud until we tell you to stop. If you make a mistake, we will let you know and ask you to start over. Please be as quick and accurate as possible. Now we would like you to begin counting backward - start at 423, and subtract 7. Continue subtracting 7's out loud until we tell you to stop."

2. Set the timer for $5 \mathrm{~min}$. Start timer.

3. If participant looks confused, have Confederate 1 say, "Would you like me to repeat that?"

4. If the participant makes a mistake, have Confederate 1 say, "That's incorrect. Please start again. 423 minus $7 . "$

5. If the participant does not start counting out loud after $30 \mathrm{~s}$, move to the next easier math problem, using same instructions as above with same prompts.

6. Use the following as the easier math problems include (in this order):

$423-5=$ ?

$95-5=?$

$66-3=?$

$6+3=?$

7. If the participant stops after each correct answer to wait for confirmation, have Confederate 1 say, "Continue counting backward by 7 . We will only correct you if you make a mistake - otherwise, keep going."

8. After 5 min, have Confederate 1 say, "Time is up. Please go next door to give your next saliva sample."

9. Escort participant back to solitary room. 


\section{Stop Protocol}

1. Empower both confederates to stop the protocol early. Both confederates are not required to consult with each other before doing so.

2. If participant cries or appears teary-eyed, becomes obviously angry or aggressive, or otherwise shows distress or if participant begins to posture in a way that appears aggressive or odd, stop protocol immediately but as normally as possible.

3. Say, "Time's up. Please go next door to give your next saliva sample."

4. Direct participant to the next room.

\section{Saliva Sample \# 2 (15 min Post-stressor Onset)}

1. When youth re-enters next room, set the timer for $15 \mathrm{~min}$.

2. Say to the participant, "That's the toughest part of your visit; thanks for doing that and we're going to talk about it in a second. It's time to collect another saliva."

3. If participant mentions TSST, say to the participant, "We can talk about that in a minute, but please do the saliva sample first.

4. Place a line to indicate $1 \mathrm{~mL}$ of saliva on a $2 \mathrm{~mL}$ cryovial, and tell participant to fill the cryovial to this line. Collect participant's saliva via passive drool through a two-inch straw into the $2 \mathrm{~mL}$ cryovial ${ }^{36}$ and freeze immediately $\left(-80^{\circ} \mathrm{C}\right)$.

5. Offer water to drink after the participant has completed the saliva sample. Do not allow the participant to keep the water; take it back when he/she is finished.

\section{Debrief}

1. Say to participant, "Okay, let's talk about the speech and math activities. You may have guessed this already, but the whole purpose of those activities was to create stress. You may have noticed that the "reviewers" weren't exactly the friendliest in the world, right?"

"Well, first of all, I want to tell you that the 'reviewers' are just research assistants. Their job is to make teens do the activities but not give any positive feedback no matter how great you do. So their behavior in there had nothing to do with your performance on those tasks - they act the same way with every single participant they meet. "

"You're probably wondering why we did that. We had a scientific reason for doing it. We are studying whether the amount of stress you experience in your daily life affects how strongly you respond to a stressful situation like this one. One point of the study is to understand why teens react differently to stress. We were measuring something called cortisol in your saliva samples as a way to see your stress response." "If you found the speech and math stressful, you're not alone! We chose making speeches and doing math because we know that even though they're safe to do, they make a lot of people nervous. Let me tell you, we needed to do those activities ourselves, and we all found it really hard! Even the scientists from Berkeley messed up the math. And we all said 'those were the longest 10 minutes of my life!' Did you feel that way?"

"A few more things I want to mention..."

[IF VIDEOTAPED] "We videotaped this for 2 reasons. The main reason is so our bosses can make sure that WE are doing the activity correctly. In the future, we may also look at the videos to get more information about people's facial expressions. It's just another way of looking at how teens respond differently to stress."

[IF NOT VIDEOTAPED] "The truth is we weren't actually videotaping you. The camera wasn't really turned on. This was another part of the acting to make it more stressful."

"The people in there weren't really writing notes about your performance. They were just writing nonsense notes as part of their acting. "We would like to ask that you please not mention this activity to anyone else who might be in the study, even if they're your best friend, brother, sister, cousin, or whatever. If other people learn about this activity before they come in for the visit, they may practice their speech at home or get nervous before they even get here, and they will have a totally different experience than you just did. We don't want that to happen. Does this make sense? For the same reason, if you talk with your parents about it, please ask them not to tell friends or family in the study."

"Do you have any questions?"

\section{Saliva Sample \#3 (30 min Post-stressor Onset)}

1. Place a line to indicate $1 \mathrm{~mL}$ of saliva on a $2 \mathrm{~mL}$ cryovial, and tell participant to fill the cryovial to this line. Collect participant's saliva via passive drool through a two-inch straw into the $2 \mathrm{~mL}$ cryovial ${ }^{36}$ and freeze immediately $\left(-80^{\circ} \mathrm{C}\right)$.

2. Set timer for $15 \mathrm{~min}$.

3. Offer water to drink after the participant has completed the saliva sample. Do not allow the participant to keep the water; take it back when he/she is finished.

\section{Saliva Sample \#4 (45 min Post-stressor Onset)}

1. Place a line to indicate $1 \mathrm{~mL}$ of saliva on a $2 \mathrm{~mL}$ cryovial, and tell participant to fill the cryovial to this line. Collect participant's saliva via passive drool through a two-inch straw into the $2 \mathrm{~mL}$ cryovial ${ }^{36}$ and freeze immediately $\left(-80^{\circ} \mathrm{C}\right)$.

2. Offer water to drink after the participant completed the saliva sample. Do not allow the participant to keep the water; take it back when he/she is finished. 


\section{Representative Results}

The sample consisted of 14 year-olds who have been a part of a richly characterized, established cohort study, the Center for the Health Assessment of Mothers and Children of Salinas (CHAMACOS). This study consists of the first cohort of 325 Mexican American families living in a farmworker community in California and followed prospectively since they were in utero. The TSST was not administered to the second CHAMACOS cohort of 300 children followed since age 9 years-old. Adolescents were screened from participating in the TSST if their full scale $I Q$ was $<70$ from a prior visit (age 12), if the adolescent was ever diagnosed with autism per maternal report, if extreme atypical behaviors shown in clinic at past visits (i.e. pronounced distrust/paranoia about study, seeing things or people who are not there - per maternal report), if their depression screener score was $\geq 3$ SDs above the mean for age and sex, and if the adolescent reported involvement in a gang fight in the previous year. These exclusion criteria were added based on over-responding initially to the TSST, but it is unclear whether these exclusion criteria made a difference in the final results. Other than these exclusion criteria, participants were enrolled no matter their psychological and physical health. In the end, 264 adolescents completed the TSST in full with either no reported problems or no problems until debrief. Table 1 lists the number of participants who were excluded and why they were excluded. There were no demographic differences between those who completed versus did not complete the TSST.

Saliva samples were stored at $-80^{\circ} \mathrm{C}$ until they were shipped on dry ice to colleagues at lowa State University (i.e., biosafety level II lab). Upon arrival, frozen saliva samples were cataloged and placed again in a $-80^{\circ} \mathrm{C}$ freezer. On the day of assay, each saliva sample was thawed and assayed within $24 \mathrm{~h}$ in duplicate using well-established highly sensitive enzyme immunoassay kits. Mean intra-assay coefficients of variation (CVs) were less than $2.25 \%$ and mean inter-assay CV was $5.13 \%$ for cortisol. The sample was reanalyzed if the CV for the duplicate measurements were greater than $7 \%$.

Descriptive statistics for the cortisol measurements along with standard deviations across each sample are provided in Table 2 and depicted in Figure 1. A $2 \times 4$ repeated measures ANOVA was run to test the effects of sex on the four saliva samples. There was a main effect for cortisol response to the TSST $(F=9.09, p<0.001)$, but sex was not a significant predictor of cortisol response to stress $(F=1.03, p=.39)$. Average body mass index was 23.53 , and was also not a significant predictor of the repeated measures cortisol ANOVA $(F=1.03, p=0.38)$. As would be expected, paired samples t-tests (provided in Table 3) revealed significant changes in cortisol from pre-stress to 15 min post-stressor onset, from $15 \mathrm{~min}$ post-stressor onset to $30 \mathrm{~min}$ post-stressor onset, and from $30 \mathrm{~min}$ post-stressor to $45 \mathrm{~min}$ post-stressor. The greatest change in cortisol occurred when comparing the pre-stress cortisol concentration to the $30 \mathrm{~min}$ post-stressor concentration, consistent with prior research (Cohen's $d=.80)^{1,3}$. Individuals who were marked as having a difficult time with the TSST tended to report heightened stress reactivity to the stressor but reported coming back to a normal state prior to the end of the study. While the 30 min post-stressor concentration was the highest concentration from these samples, the $45 \mathrm{~min}$ post-stressor cortisol concentration suggests that individuals began to recover from the stressor, as the 45 min post-stressor concentration was significantly lower than the 30 min concentration (see Table 3 ).

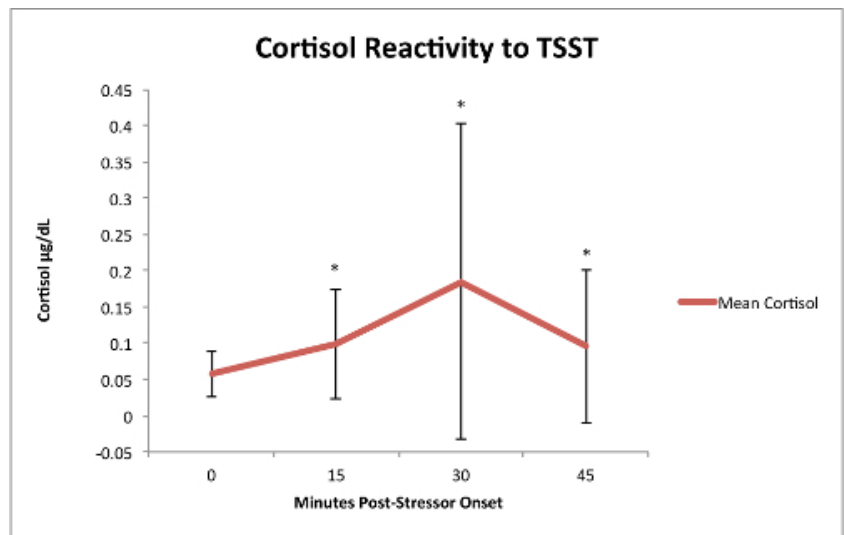

Figure 1: Cortisol Reactivity to the TSST. Cortisol reactivity means plotted with standard deviations. Please click here to view a larger version of this figure.

\begin{tabular}{|l|c|}
\hline Reason for Exclusion & N \\
\hline Started but stopped early & 10 \\
\hline Refused to participate & 7 \\
\hline Screened out of project & 13 \\
\hline Skipped due to project related issues* & 8 \\
\hline Total & 38 \\
\hline \multicolumn{2}{|l|}{ Not enough study personnel present to administer TSST. }
\end{tabular}

Table 1: Reasons for Exclusion. Participants were excluded from the study if they started the TSST but stopped early, refused to participate, were screened out of the project, or were skipped due to project related issues. 


\begin{tabular}{|l|r|r|}
\hline Saliva Sample & Mean Cortisol & SD \\
\hline Prc-stress (0 min post-strcssor onsct) & 0.058 & 0.032 \\
\hline 15 min post-stressor onset & 0.099 & 0.076 \\
\hline 30 min post-stressor onset & 0.185 & 0.217 \\
\hline 45 min post-stressor onset & 0.096 & 0.106 \\
\hline
\end{tabular}

* Cortisol Concentration $(\mu \mathrm{g} / \mathrm{dL}$ )

*SD $=$ Standard Deviations

Table 2: Mean Cortisol Concentrations and Standard Deviations. Cortisol increased from samples 1-3, and then declined by sample 4.

\begin{tabular}{|l|r|r|r|l|}
\hline Sample Comparisons & Mean Change & SD & t -test & Significance \\
\hline Pre-stress to 15 min post & 0.041 & 0.068 & 4.287 & $p<.001$ \\
\hline 15 min to 30 min post & 0.086 & 0.158 & 3.819 & $p<.001$ \\
\hline Pre-stress to 30 min post & 0.127 & 0.213 & 4.202 & $p<.001$ \\
\hline 30 min to 45 min post & -0.086 & 0.158 & -3.819 & $p<.001$ \\
\hline Pre-stress to 45 min post & 0.038 & 0.106 & 2.502 & $p<.01$ \\
\hline
\end{tabular}

Table 3: Paired Samples t-tests. Compares samples to one another in cortisol concentration ( $\mu \mathrm{g} / \mathrm{dL})$ and standard deviations (SD) for cortisol change. Significant increases in cortisol were observed from the baseline sample to post-stress samples.

\section{Discussion}

The current study sought to confirm the efficacy of a modified version of the TSST with vulnerable youth. The CHAMACOS project presented the optimal sample to test the effectiveness of a modified contextually appropriate TSST, as it is both unique (Mexican American samples are often excluded from research) and represents a sample of youth who have experienced significant early life adversity ${ }^{37}$. Results confirm the success of the modified TSST in provoking a significant increase in cortisol from pre-stress to post-stress samples, as well as a normal relative recovery from the TSST by the time the last saliva sample was collected. This study provides a valuable tool to the field through both the contextually appropriate protocol of the TSST as well as an exploration of the factors that influenced the modifications of the original TSST.

There are several critical steps for the set up and administration of the TSST that must be attended to. First, both confederates need to wear white lab coats, sit behind a table, and have a video camera recording and pointed towards where the participant will be standing before the participant is ushered into the room. The participant stands throughout the entire TSST, while the committee sits. Second, there needs to be 1 confederate of each sex, and the confederates need to be of similar race/ethnicity to the participant, and appearing of similar age as the participant (this study used slightly older youth who looked similar in age to the participants). Third, confederates must demonstrate flat affect, with slight boredom throughout the protocol. Confederates are to look at the participant as much as possible without showing interest during the TSST. Confederate 2 writes notes, whereas Confederate 1 is responsible for administering prompts and instructions.

It should be noted that conducting the TSST in an emotionally "neutral" state takes skill and practice. Some individuals may not be appropriate for the confederate role if they are not able to maintain a neutral face. Confederates in this study were hand-selected for being able to maintain the neutral stance during the entire TSST. However, should a participant become tearful, sad, or aggressive, these personnel need to know how and when to intervene to stop the protocol. The integrity of the TSST also needs to be assessed periodically throughout the study to ensure there is no drift in the emotional demeanor or script. In this study, weekly check-ins were provided to confederates when they were able to troubleshoot any unusual occurrences with experts in TSST administration and check in with questions. TSST "refreshers" were also provided every few months to retrain confederates on the TSST. Videos were recorded during the TSST for participants whose mothers provided consent, and these videos were reviewed to ensure veracity to the TSST script. Finally, some researchers may think that participants who expressed betrayal or anger in response to the TSST will have high cortisol responses. However, this study ruled out this notion in the data, as it turned out that these youths were most likely to be non-responders (i.e., individuals whose cortisol either decreased or remained stable across the task) as assessed via their cortisol reactions. Thus, strictly adhering to a sensitive protocol did not undermine the overall reactivity rates, but rather ensured that the stressor's challenge was titrated to the appropriate level for these youth. The standard TSST protocol would thus have been inappropriate both ethically but also scientifically as attrition, nonresponse, and "giving up" was a predominant response.

After running a few participants through the original TSST, the original TSST was modified to better match the contextual factors of this population due to non-optimal responses (i.e., crying, disengaging from the task, aggressive posturing). Study personnel consulted with numerous researchers who have used the TSST with vulnerable populations (e.g., orphanages, juvenile justice settings, abused populations) but none of the modifications fit this study's needs perfectly - some veered too far from the protocol and we were concerned that these changes would not elicit a cortisol response ${ }^{12,27}$. Thus, three primary modifications were applied to the TSST in our study, and the modifications and motivations for these modifications are illuminated below.

First, it is important not to alienate participants. Administration of a stress task was important to this study, yet it was imperative that participants were not lost to attrition, as the youth had been followed longitudinally since their mothers became pregnant with them. In this study, participants were debriefed immediately after the TSST in order to ensure that the stress ceased for participants after the conclusion of the TSST. For this sample, the staff administering the debriefing needed to be someone as unfamiliar as possible but still empathic, without an emotional connection to the youth. While that may seem counterintuitive, when staff who were familiar and emotionally connected to the youth debriefed them on the TSST, the youth tended to have more proclivity towards rumination over the TSST and talking more about it, which sometimes ended with crying or feelings of resentment towards being "tricked." Unfamiliar staff administering the debriefing helped ensure that we did not sever the longitudinal connections that the cohort had made with the familiar staff by implementing the TSST. This is noted as important within the Latino community but is something that many longitudinal studies that develop a strong rapport with participants should consider. Using unfamiliar staff also reduced the likelihood that the debrief served to further stress the youth, given the more optimal behavioral responses we observed using unfamiliar staff. While debriefing before the collection of two more saliva samples may concern researchers using this modification, it should be noted that this study observed the prototypical cortisol response pattern after the conclusion of the TSST (with 
the highest concentration coming $15 \mathrm{~min}$ after the conclusion of the stressor, and recovery 30 min after the conclusion of the stressor). This modification was made to be more culturally sensitive to the youth whom may have continued to have a stress response after the TSST due to their vulnerable state.

Second, the TSST protocol needs to be flexible to the individual factors of the participant. The Mexican American youth in this study reside in low-resourced and impoverished agricultural communities in the Salinas Valley. CHAMACOS families labor under difficult conditions, specifically low-wage, intermittent seasonal employment and long working hours that circumvent opportunities for positive familial interactions during a time of rapid and salient adolescent development. Stressors associated to low SES/poverty in the cohort contribute to food insecurity, overcrowded housing, substandard living conditions, and inadequate medical services to address cognitive disabilities and mental health needs. Compounding this is that youth in the Salinas Valley are disproportionately exposed to family turmoil and youth violence, which spill into local schools and neighborhoods alike. These risk factors become increasingly accentuated as youth either learn to adapt to rapidly changing social and economic challenges or gradually become sensitized (or in some cases desensitized) to the unpredictable nature of their daily lives. One example of making the TSST more flexible for this population was to vary the difficulty of the math section. Given that this study was privy to previous cognitive testing and had knowledge of environmental pathogen exposure, which may impact $I Q^{38}$, it was clear that the "one size fits all" math task typically associated with the TSST was not going to accommodate this population. Following other stressors ${ }^{39,40}$, math difficulty was therefore titrated to be challenging but not impossible for the youth.

Third, the TSST protocol needs to recognize and build upon the high sense of community and social connection within certain cultural settings. It is important to use confederates that youth participants connect with, so this study recruited confederates that were slightly older than the participants so as to appear of similar age and ethnicity and came from the same community so SET came from "their peers". Conversely, too much social connection was problematic. It is important to ensure that confederates are appropriately neutral and that confederates were unknown to the participants. This was critical for the current study because although the Salinas Valley is a relatively large region, the youth community can be very small and interconnected. Thus, it can be useful to ensure before the TSST commences that the participants and confederates do not know each other. If the participant or confederate recognized the other in this study, the TSST was run with just one confederate. The fact that this study's confederates came from the same community is important to note in this context (Salinas), which is far from a university setting and difficult when finding staff who can work an hour or two at a time. This study's field office researchers were very savvy and highly trained regarding research, which should not be taken for granted in terms of maintaining protocol.

While these results are promising for validating the use of a modified TSST with vulnerable adolescents, more research is needed to confirm the replicability of these effects across different samples. First, while this project was fortunate to be able to access such a unique and vulnerable sample of Mexican American adolescents, these results need to be replicated in other vulnerable ethnicities in order to confirm that this modified TSST is potent enough to elicit a cortisol response in other populations. Second, this sample was limited to 14-year-olds. While the narrow sampling controlled for the effect of age on TSST response in this sample, future research could attempt to replicate these effects in different adolescent and child ages, and perhaps across pubertal development. Finally, it should be noted that data was not collected from a comparison group utilizing a non-modified TSST, as it would be unethical with this sample given the high rate of poor behavioral and emotional responses we were experiencing in the beginning with a less modified version of the TSST.

There are also limitations of the TSST technique in general. As mentioned earlier, it takes significant practice for confederates to maintain neutral stance throughout the entire TSST. At least 3 people are needed to run the TSST appropriately ( 2 confederates and 1 experimenter), and the TSST runs better if these people are unknown to the participant. Thus, this protocol is easier to run in college campus settings where undergraduates can work in the lab 1-2 $\mathrm{h}$ at a time, but less easy in remote settings or in settings where confederates are being paid as a job (as they are only paid for $\sim 1 \mathrm{~h}$ of work per TSST). This technique could also be a hurdle for longitudinal studies with personnel that work on the project over time - these well-known personnel should not be the ones administering the TSST. Also, there is significant overhead in terms of maintaining the integrity of the TSST over time - experts are needed to review the video tapes of the TSST, maintain weekly check ins with the confederates, and provide periodical "refresher" sessions to ensure there is no drift in the technique. Further, the TSST is difficult to do repeatedly for a given participant, as it relies on minor deception and participants may habituate to the technique. The speech and math portions are not counterbalanced, which would be more optimal for other methodologies such as autonomic nervous system functioning. There are no parts to the TSST that are non-stressful, so it requires another session (i.e., friendly TSST or a placebo TSST) or something else (i.e., a basal day) to have a non-stress comparison. Finally, a non-lab-based stressor might be more effective (i.e., karaoke, roller coasters, or haunted houses) or more ecological ${ }^{41}$.

This study provides the foundation for future research to utilize a modified TSST with vulnerable youth. Future longitudinal studies seeking to retain participants across multiple waves of data collection may also need to modify their approach utilizing some of these modifications so as to limit attrition. Further, this study shows that it is indeed possible to create a modified version of the TSST that is culturally sensitive while still titrating stress reactivity to an appropriate level to capture individual differences in cortisol responsivity.

\section{Disclosures}

There are no competing financial interests.

\section{Acknowledgements}

The authors would like to thank the parents and adolescents who participated in the CHAMACOS study. We also wish to thank the CHAMACOS staff who worked on the study, especially Katherine Kogut, M.S., for all the work she did as project manager for this study. The authors would also like to acknowledge the National Institute of Drug Abuse grant 1R01DA035300. 


\section{References}

1. Kirschbaum, C., Pirke, K. M., \& Hellhammer, D. H. The 'Trier Social Stress Test'--a tool for investigating psychobiological stress responses in a laboratory setting. Neuropsychobiology. 28 (1-2), 76-81 (1993).

2. Yim, I. S., Quas, J. A., Rush, E. B., Granger, D. A., \& Skoluda, N. Experimental manipulation of the Trier Social Stress Test-Modified (TSSTM) to vary arousal across development. Psychoneuroendocrinology. 57 61-71 (2015).

3. Dickerson, S. S., \& Kemeny, M. E. Acute stressors and cortisol responses: a theoretical integration and synthesis of laboratory research. Psychol Bull. 130 (3), 355-391 (2004).

4. Nelson, E. E., Leibenluft, E., McClure, E. B., \& Pine, D. S. The social re-orientation of adolescence: a neuroscience perspective on the process and its relation to psychopathology. Psychol Med. 35 (2), 163-174 (2005).

5. Nelson, H. J., Kendall, G. E., \& Shields, L. Neurological and biological foundations of children's social and emotional development: an integrated literature review. J Sch Nurs. 30 (4), 240-250 (2014).

6. Crone, E. A., \& Dahl, R. Understanding adolescence as a period of social-affective engagement and goal flexibility. Nature. $13636-650$ (2012).

7. Rith-Najarian, L. R., McLaughlin, K. A., Sheridan, M. A., \& Nock, M. K. The biopsychosocial model of stress in adolescence: self-awareness of performance versus stress reactivity. Stress (Amsterdam, Netherlands). 17 (2), 193-203 (2014).

8. Ho, S. S., Konrath, S., Brown, S., \& Swain, J. E. Empathy and Stress Related Neural Responses in Maternal Decision Making. Front Neuro. 8 (2014).

9. Wager, T. D. et al. Brain mediators of cardiovascular responses to social threat: Part I: Reciprocal dorsal and ventral sub-regions of the medial prefrontal cortex and heart-rate reactivity. Neurolmage. 47 (3), 821-835 (2009).

10. Bosch, J. A. et al. A general enhancement of autonomic and cortisol responses during social evaluative threat. Psychosom Med. 71 (8), 877-885 (2009).

11. Dickerson, S. S., Mycek, P. J., \& Zaldivar, F. Negative social evaluation, but not mere social presence, elicits cortisol responses to a laboratory stressor task. Health Psychol. 27 (1), 116-121 (2008).

12. Het, S., Rohleder, N., Schoofs, D., Kirschbaum, C., \& Wolf, O. T. Neuroendocrine and psychometric evaluation of a placebo version of the 'Trier Social Stress Test'. Psychoneuroendocrinology. 34 (7), 1075-1086 (2009).

13. Bouma, E. M., Riese, H., Ormel, J., Verhulst, F. C., \& Oldehinkel, A. J. Adolescents' cortisol responses to awakening and social stress; effects of gender, menstrual phase and oral contraceptives. The TRAILS study. Psychoneuroendocrinology. 34 (6), $884-893$ (2009).

14. Westenberg, P. M. et al. A prepared speech in front of a pre-recorded audience: subjective, physiological, and neuroendocrine responses to the Leiden Public Speaking Task. Biol Psychol. 82 (2), 116-124 (2009).

15. Kelly, O., Matheson, K., Martinez, A., Merali, Z., \& Anisman, H. Psychosocial stress evoked by a virtual audience: relation to neuroendocrine activity. Cyberpsychol Behav. 10 (5), 655-662 (2007).

16. Wadiwalla, M. et al. Effects of manipulating the amount of social-evaluative threat on the cortisol stress response in young healthy women. Stress. 13 (3), 214-220 (2010).

17. Schwabe, L., Haddad, L., \& Schachinger, H. HPA axis activation by a socially evaluated cold-pressor test. Psychoneuroendocrinology. (2008).

18. Taylor, S. E. et al. Effects of a supportive or an unsupportive audience on biological and psychological responses to stress. $J$ Pers Soc Psychol. 98 (1), 47-56 (2010).

19. Taylor, S. E. et al. Neural bases of moderation of cortisol stress responses by psychosocial resources. J Pers Soc Psychol. 95 (1), $197-211$ (2008).

20. Britton, W. B., Shahar, B., Szepsenwol, O., \& Jacobs, W. J. Mindfulness-based cognitive therapy improves emotional reactivity to social stress: results from a randomized controlled trial. Behav Ther. 43 (2), 365-380 (2012).

21. Brown, K. W., Weinstein, N., \& Creswell, J. D. Trait mindfulness modulates neuroendocrine and affective responses to social evaluative threat. Psychoneuroendocrinology. 37 (12), 2037-2041 (2012).

22. Creswell, J. D. et al. Affirmation of personal values buffers neuroendocrine and psychological stress responses. Psychol Sci. 16 (11), $846-851$ (2005).

23. Lam, S., Dickerson, S. S., Zoccola, P. M., \& Zaldivar, F. Emotion regulation and cortisol reactivity to a social-evaluative speech task. Psychoneuroendocrinology. 34 (9), 1355-1362 (2009).

24. Pilgrim, K., Marin, M. F., \& Lupien, S. J. Attentional orienting toward social stress stimuli predicts increased cortisol responsivity to psychosocial stress irrespective of the early socioeconomic status. Psychoneuroendocrinology. (2009).

25. Cavanagh, J. F., \& Allen, J. J. Multiple aspects of the stress response under social evaluative threat: an electrophysiological investigation. Psychoneuroendocrinology. 33 (1), 41-53 (2008).

26. Denson, T. F., Fabiansson, E. C., Creswell, J. D., \& Pedersen, W. C. Experimental effects of rumination styles on salivary cortisol responses. Motivation Emotion. 33 42-48 (2009).

27. Wiemers, U. S., Schoofs, D., \& Wolf, O. T. A friendly version of the trier social stress test does not activate the HPA axis in healthy men and women. Stress. 16 (2), 254-260 (2013).

28. Halpern, C. T., Campbell, B., Agnew, C. R., Thompson, V., \& Udry, J. R. Associations between stress reactivity and sexual and nonsexual risk taking in young adult human males. Horm Behav. 42 (4), 387-398 (2002).

29. Gunnar, M. R., Talge, N. M., \& Herrera, A. Stressor paradigms in developmental studies: what does and does not work to produce mean increases in salivary cortisol. Psychoneuroendocrinology. 34 (7), 953-967 (2009).

30. Gunnar, M. R., Frenn, K., Wewerka, S. S., \& Van Ryzin, M. J. Moderate versus severe early life stress: associations with stress reactivity and regulation in 10-12-year-old children. Psychoneuroendocrinology. 34 (1), $62-75$ (2009).

31. Martel, F. L. et al. Salivary cortisol levels in socially phobic adolescent girls. Depress Anxiety. 10 (1), $25-27$ (1999).

32. Mendes, W. B., Blascovich, J., Lickel, B., \& Hunter, S. Challenge and threat during social interactions with white and black men. Pers Soc Psychol Bull. 28 939-952 (2002).

33. Mendes, W. B., Gray, H. M., Mendoza-Denton, R., Major, B., \& Epel, E. S. Why egalitarianism might be good for your health: physiological thriving during stressful intergroup encounters. Psychol Sci. 18 (11), 991-998 (2007). 
34. Page-Gould, E., Mendoza-Denton, R., \& Tropp, L. R. With a little help from my cross-group friend: reducing anxiety in intergroup contexts through cross-group friendship. J Pers Soc Psychol. 95 (5), 1080-1094 (2008).

35. Felitti, V. J. et al. Relationship of childhood abuse and household dysfunction to many of the leading causes of death in adults. The Adverse Childhood Experiences (ACE) Study. Am J Prev Med. 14 (4), 245-258 (1998).

36. Schwartz, E. B., Granger, D. A., Susman, E. J., Gunnar, M. R., \& Laird, B. Assessing salivary cortisol in studies of child development. Child Dev. 69 (6), 1503-1513. (1998).

37. Alkon, A. et al. Prenatal adversities and Latino children's autonomic nervous system reactivity trajectories from 6 months to 5 years of age. PLoS One. 9 (1), e86283 (2014).

38. Bouchard, M. F. et al. Prenatal exposure to organophosphate pesticides and IQ in 7-year-old children. Environ Health Perspect. 119 (8), 1189-1195 (2011).

39. Pruessner, J. C. et al. Stress regulation in the central nervous system: evidence from structural and functional neuroimaging studies in human populations - 2008 Curt Richter Award Winner. Psychoneuroendocrinology. 35 (1), 179-191 (2010).

40. Dedovic, K. et al. The Montreal Imaging Stress Task: using functional imaging to investigate the effects of perceiving and processing psychosocial stress in the human brain. J Psychiatry Neurosci. 30 (5), 319-325 (2005).

41. Shirtcliff, E. A., Peres, J. C., Dismukes, A. R., Lee, Y., \& Phan, J. M. Riding the physiological roller coaster: Adaptive significance of cortisol stress reactivity to social contexts. J Pers Disord. 28 (1), 65-76 (2014). 